\title{
LRH1 promotes tumor cell proliferation and migration, and is correlated with poor prognosis in ovarian cancer
}

\section{Wenzhou Sun}

Tumor Hospital of Harbin Medical University

Qingtao Shi

Tumor Hospital of Harbin Medical University

Jiaxin Li

Tumor Hospital of Harbin Medical University

Jinmeng Li

Tumor Hospital of Harbin Medical University

Libo Yu ( $\nabla$ ylb13030082698@sina.com )

harbin medical univeristy cancer hospital https://orcid.org/0000-0002-9111-8412

\section{Primary research}

Keywords: Liver receptor homolog 1 (LRH1), ovarian cancer, proliferation, metastasis, prognosis

Posted Date: June 24th, 2020

DOI: https://doi.org/10.21203/rs.3.rs-26942/v2

License: (c) (1) This work is licensed under a Creative Commons Attribution 4.0 International License. Read Full License 


\section{Abstract}

Background Liver receptor homolog 1 (LRH1) plays a vital role in several human cancers, but its role in ovarian cancer $(\mathrm{OC})$ remains unclear. We aimed to explore the functions of LRH1 and its clinical relevance.

Methods LRH1 expression was evaluated by immunohistochemistry and reverse transcription quantitative polymerase chain reaction (RT-qPCR). The effects of LRH1 on tumor cell proliferation, migration and epithelial-mesenchymal transition (EMT) were evaluated in vitro. Furthermore, bioinformatics analysis was applied to predict the functions of LRH1.

Results RT-qPCR showed that LRH1 mRNA expression was intense in the invasive lesions $(P<0.05)$. LRH1 overexpression was extremely related with elevated International Federation of Gynecology and Obstetrics ( $F I G O)$ stage $(P=0.001)$, lymph node metastasis $(P=0.011)$, and peritoneal metastasis $(P=$ 0.001). Furthermore, $L R H 1$ expression was an independent prognostic index for disease-free survival in patients with OC $(P=0.041)$. LRH1 overexpression $(P=0.011)$, FIGO stage $(P<0.001)$, and ascites $(P=0.015)$ independently affected peritoneal metastasis in patients with OC. LRH1 knockdown significantly inhibited the proliferation, migration, and EMT of human $O C$ cells $(P<0.05)$. Bioinformatics analysis indicated that the functions of LRH1 were associated with the PRC1 complex, nuclear ubiquitin ligase complex, and Polycomb-group (PcG) proteins.

Conclusions This study provides evidence of the predictive value of $\mathrm{LRH} 1$ on peritoneal metastasis and poor outcome and highlights the potential role of LRH1 as a biomarker for the targeted therapy of OC. Furthermore, LRH1 promotes OC cell proliferation, migration, and EMT in vitro, and its functions may be associated with PRC1 complex, nuclear ubiquitin ligase complex, and PcG proteins.

\section{Background}

Ovarian cancer (OC) is the most lethal gynecologic malignancy among women. An estimate of 21,750 new cases of OC will be diagnosed and 13,940 women will die of OC in the United States in 2020 according to the American Cancer Society [1]. The standard treatment for advanced OC is primary cytoreductive surgery followed by adjuvant chemotherapy using a platinum analog plus paclitaxel [2-4]. Poly ADP ribose polymerase inhibitors (PARP) used in the management of epithelial ovarian cancer (EOC) have renewed the hope of patients with EOC. Unfortunately, the clinical effects of PARPi therapy in patients with $\mathrm{OC}$ are less favorable because of PARPi resistance [5-8]. The prognosis for the advanced stage of $\mathrm{OC}$ remains poor even though a novel technology has been applied for the diagnosis and treatment of $\mathrm{OC}$ [9]. We have gained insight into $\mathrm{OC}$ in recent years, although advancing knowledge has been previously hindered by substantial disease heterogeneity and uncertainties the origin of tumor tissues. Therefore, the identification of an ideal biological factor that predicts the malignant biological behavior and prognosis of $\mathrm{OC}$ will be helpful. 
Liver receptor homolog 1 (LRH1), also known as nuclear receptor subfamily 5 group A member 2 NR5A2, participates in various biological processes, such as liver and pancreas differentiation, steroidogenesis in childhood, cholesterol/bile acid homeostasis, and tumor progression [10,11]. Previous studies validated that the overexpression of LRH1 may induce the resistance of breast cancer to chemotherapy [12,13], the metastasis of pancreas cancer [14], the poor prognosis of colon cancer [15] and non-small cell lung cancer [16], and the proliferation of hepatoblastoma [17]. However, LRH1 expression has not yet been reported in patients with $\mathrm{OC}$.

Thus, we aimed to explore the status of $\mathrm{LRH} 1$ protein expression in $\mathrm{OC}$ and its clinical relevance. The functions of LRH1 were predicted using bioinformatics analysis.

\section{Materials And Methods}

\section{Human tissue samples and clinical data}

OC tumor tissue samples were obtained from Harbin Medical University Cancer Hospital between January 2009 and December 2011 from 133 patients with OC who underwent cytoreductive surgery without any prior treatment for cancer, such as chemotherapy or radiation. The International Federation of Gynecology and Obstetrics (FIGO) staging system was applied to assess tumor stage [18]. Histological grades were based on the World Health Organization's Histological Grading System for tumors [19]. Furthermore, the subjects underwent lymph node dissection to assess lymph node status. Twenty-five patients with normal ovaries who underwent hysterectomy with oophorectomy in our hospital for benign uterine disease during the same period were also included.

Approval from the Medical Ethics Committee of Harbin Medical University Cancer Hospital was obtained for the purpose of research. The 133 patients with OC were followed up for survival analyses until death or until the study closing date (January 2018).

\section{Immunohistochemical (IHC) staining and evaluation}

133 tissue blocks of $\mathrm{OC}$ and 25 of normal ovary tissues were cut by a microtome into $4-\mu \mathrm{m}$ sections and affixed onto the slide. After dewaxing in xylene and rehydrating through graded alcohol concentrations, the sections were incubated with $0.3 \%$ hydrogen peroxide for 10 minutes at room temperature to block endogenous peroxidase, and then the sections were incubated with anti-LRH1 antibody (1:100, Abcam, ab223211) overnight at $4^{\circ} \mathrm{C}$. After washing in phosphate-buffered saline (PBS), all sections were incubated with secondary antibodies (R\&D Systems, NL004) at room temperature for 20 min and then treated with 3,3'-diaminobenzidine tetrahydrochloride (Dako, Hamburg, Germany), following by counterstaining with hematoxylin.

Protein expression level of $\mathrm{LRH} 1$ was scored by evaluating the percentage of the positive staining areas of tumor cells together with intensity of staining. The former was scored as follows [20]: $0,<0 \% ; 1,1-10 \%$; $2,11-50 \% ; 3,51-70 \%$ and $4, \geq 70 \%$. The latter was scored as follows: 0 , negative staining; 1 , weak 
staining; 2, moderate staining; and 3, intense staining. The final expression level of LRH1 was semiquantitatively evaluated according to the sum of the scores for the percentage of positively stained tumors cells and intensity scores (0-7) in which the final staining scores of $0-3$ and 4-7 were considered to be low and high expression, respectively.

The IHC staining on each slide was scored in twice independently by two pathologists who were sophisticated in evaluating IHC and blinded with the clinicopathological information.

\section{Cell culture}

The human OC cell lines SKOV3 and OVCAR3 (ATCC) were employed. Both the cell lines have been approved by short tandem repeat (STR) profiling. Cells were cultured at $37{ }^{\circ} \mathrm{C}$ in a humidified atmosphere containing 5\% CO2 in DMEM complemented with 10\% Fetal bovine serum (FBS) (Hyclone, USA) and antibiotics (penicillin and streptomycin). Cells were passaged when they reached $80 \%$ confluence.

\section{Western blotting analysis}

The cell lysates were both centrifuged at $13000 \mathrm{rpm}$ (at $4^{\circ} \mathrm{C}$ for 5 minutes). After that $10 \%$ sodium dodecyl sulfate polyacrylamide gel electrophoresis (SDS-PAGE) were used to segregate them, and then transferred onto polyvinylidene difluoride (PVDF) membranes (Millipore, Bedford, MA, USA). The filters were blocked with blocking buffer (Sangon Biotech) for 45min.Membranes were incubated with Primary antibodies anti-LRH1 (Abcam, ab223211), anti-E-cadherin (Abcam, ab1416), anti-N-cadherin (Abcam, ab18203), anti-Vimentin (Abcam, ab92547), anti- $\beta$-catenin (Cell Signaling Technology, D10A8)) at $4^{\circ} \mathrm{C}$ overnight, and with secondary antibodies at 1:5000 dilution at room temperature for $2 \mathrm{~h}$, following by incubating with $\beta$-actin (Abcam, ab179467) atroom temperature for 1.5h.

\section{RNA extraction and reverse transcription (RT)-qPCR}

The TRIzol ${ }^{T M}$ LS Reagent (Invitrogen ${ }^{T M}$ ) was used to extract total RNA from tissues and cells, according to the manufacturer's protocol, followed by complementary DNA synthesis using a PrimeScript RT reagent Kit with gDNA Eraser (Takara). Amplification was subsequently carried out using the GeneAmp ${ }^{\circledR}$ PCR System 9700 (Applied Biosystems, USA). Real-time PCR was performed using LightCycler ${ }^{\circledR} 480 \otimes$ Realtime PCR Instrument (Roche, Swiss). The mRNA expression levels of LRH-1 were normalized to the mRNA levels of GAPDH, which was used as an internal control. The $2^{-\Delta \Delta C t}$ method was used to quantify the mRNA expression levels [21].

\section{Establishment of stable short hairpin (sh)RNA-mediated LRH-1 knockdown ovarian cancer cell lines.}

shRNA-induced knockdown of LRH-1 expression was achieved using the lentiviral expression system. The plasmid used in the present study were pPLK/GFP+Puro-NR5A2 (Genomeditech, China). Viral particles

were generated by co-transfecting 293T cells (ATCC) with the shRNAs and the GM easy ${ }^{\text {TM }}$ Lentivirus Packaging kit (Genomeditech, China), which contains packaging plasmids and a transfection reagent according to the manufacturer's protocol. Subsequently, the shRNA viral particles transfected SKOV3 and 
OVCAR3 cells with $4 \mu \mathrm{g} / \mathrm{mL}$ polybrene (Sigma-Aldrich), and stable cell lines were established after 10 days of puromycin $(2 \mu \mathrm{g} / \mathrm{ml})$ selection. Knockdown was confirmed using RT-qPCR or immunoblotting. The selected cell lines were routinely cultured in puromycin-containing media until 2 days prior to experimentation.

\section{Transwell assay}

For migration assays, infected OVCAR3 cells $\left(1 \times 10^{5}\right.$ in $200 \mu \mathrm{L}$ of serum-free DMEM medium) and SKOV3 cells $\left(1 \times 10^{5}\right.$ in $200 \mu \mathrm{L}$ of serum-free DMEM medium) were seeded into the upper chamber of transwell plates in a 24-well format with $8 \mu \mathrm{m}$ diameters (Corning Costar, USA). Then, $600 \mu \mathrm{L}$ of medium containing $10 \%$ FBS was added to the bottom chamber as a chemoattractant. After 24 hours of culture, cells were fixed with methanol and stained with Crystal Violet solution. The remaining cells were removed from the top of the permeable membrane using a cotton swab. Then, cells that migrated through the upper chamber were counted in 4 random fields under a light microscope (NIKON INSTRUMENTS \SHANGHAIखCO.,LTD).

\section{Wound healing assay}

OVCAR3 $\left(1 \times 10^{5}\right.$ cells $)$ and SKOV3 cells $\left(1 \times 10^{6}\right.$ cells) were seeded in 6 -well cell culture plates and incubated for 24 hours at $37^{\circ} \mathrm{C}$. After achieving confluence, the cellular layer in each plate was scratched using a plastic pipette tip. The migration of the cells at the edge of the scratch was analyzed at 0 and 24 hours, when microscopic images of the cells were captured.

\section{Cell counting kit-8 (CCK-8)}

SKOV3 and OVCAR3 cells were plated in flat-bottom 96-well plates (1500 and 1000 cells/well) and supplemented with $100 \mu \mathrm{L}$ DMEM medium with $10 \% \mathrm{FBS}$ per well. After incubation at $37^{\circ} \mathrm{C}$ in a humidified incubator with 5\% CO2 for 2, 24, 48, 72, and 96 hours, respectively, $10 \mu \mathrm{L}$ of CCK-8 (Yeasen Biotech Co., Ltd.) was added to each well. Then, after 1 hours of culture, colorimetric analysis was performed on a microplate reader (Thermo Scientific ${ }^{\text {TM }}$ Varioskan ${ }^{T M}$ LUX) at a wavelength of $450 \mathrm{~nm}$. The assay was performed using six replicates.

\section{Statistical analysis}

The chi-square test was used to analyze the statistical differences of the clinicopathologic variables. Overall survival (OS) and disease-free survival (DFS) were evaluated by the Kaplan-Meier method and logrank test. The Cox proportional hazards model was used to estimate the independent prognostic factors for survival. Logistic regression was performed for multivariate analysis of the association between LRH1 expression and intraperitoneal metastasis. Student's t-test were used to analyze the differences between the experimental and control groups. A two-sided $\mathrm{P}<0.05$ was considered significant.

\section{UALCAN}


UALCAN (http://ualcan.path.uab.edu) is an interactive web resource based on TCGA database, which can be used to analyze relative transcriptional expression of potential genes of interest between tumor and normal samples and association of the transcriptional expression with relative clinicopathologic parameters [22]. In this study, we use UALCAN to explore the association of LRH1 mRNA expressions in ovarian cancer tissues and clinicopathologic parameters. Students' $t$ test was applied to compared the difference of mRNA expression and $p<0.01$ was considered as statically significant.

\section{GEPIA}

GEPIA (http://gepia.cancer-pku.cn/) is a web server to analyze cancer and normal gene expression and interaction [23]. In this study, we use GEPIA to obtain a series of genes that have similar expression patterns with LRH1 in ovarian cancer.

\section{Metascape}

The Metascape (http://metascape.org/) is an online analytical tool, which facilitates gene annotation integration, functional enrichment, interactome analysis [24]. In the present study, Metascape was applied to gene-enrichment analysis of genes that have similar expression patterns with LRH1 in ovarian cancer.

\section{Results}

\section{Expression level of LRH1 in OC tissues}

RT-qPCR was carried out to determine the difference in the LRH1 mRNA expression between OC and normal ovarian tissues (Figure 1). No discrepancy was observed in the LRH1 mRNA expression between normal tissues and OC tissues without metastasis (T1) $(P>0.05)$. However, the LRH1 mRNA expression in OC tissues with metastasis presents (T2) was higher than that in T1 $(P=0.017)$. Moreover, LRH1 mRNA expression was more intense in the invasive foci (M) than in the situ foci (T2) among the same patients $(P=0.034)$.

$\mathrm{IHC}$ revealed that the expression of $\mathrm{LRH} 1$ was scarcely detectable in normal tissues but obvious in $\mathrm{OC}$ tissues (Figure 2). As shown in Figure 2B, LRH1 was observed in the nucleus and cytoplasm of tumor cells but was localized in the cytoplasm of cells in normal tissues. Among the $133 \mathrm{OC}$ specimens examined, 59 (44.4\%) had low LRH1 expression and 74 (55.6\%) had high LRH1 expression. Furthermore, the patients with $\mathrm{OC}$ who had high LRH1 expression comprised $28.6 \%$ of patients with $\mathrm{FIGO} \mathrm{I/II} \mathrm{and}$ $62.9 \%$ of patients with FIGO III/IV.

LRH1 mRNA expression patterns were further measured by UALCAN. The data resources of UALCAN were based on The Cancer Genome Atlas database. As shown in Figure 4A, LRH1 mRNA expression was upregulated in stage II adn stage IV OC tissues compared with stage I and stage III OC tissues, but the difference was not statistically significant. 
Table 1 summarizes the association between LRH1 expression and clinicopathological variables in OCs. LRH1 expression was significantly related to FIGO stage $(P=0.001)$, lymph node metastasis $(P=0.011)$, and intraperitoneal metastasis $(P=0.001)$. LRH1 expression was not related to age, histological type, histologic grade, residual disease, ascites, and serum CA-125 level $(P>0.05)$.

\section{Increased LRH1 expression in OC predicts unfavorable prognosis and recurrence}

Log-rank test showed that patients with low LRH1 expression had a significantly better OS $(P=0.007)$ and DFS $(P=0.001)$ compared with patients with high LRH1 (Figure 3). FIGO stage (both $P<0.001$ ), histologic grade $(P=0.0090 .028$, respectively), residual disease $(P=0.022$ and 0.026 , respectively), lymph node metastasis (both $P<0.001)$, and intraperitoneal metastasis $(P=0.0010 .001$, respectively) were correlated with the prognosis of patients with OC regardless of low and high LRH1 expression (Table 2). Multivariate Cox regression analysis showed that high LRH1 expression was considered an independent prognostic marker for the DFS of patients with OC instead of OS ( $P=0.041$; Table 2).

\section{Increased LRH1 expression as indicator of intraperitoneal and lymph node metastases in OC}

The traditional clinicopathological parameters of intraperitoneal metastasis were first appraised to evaluate the relationship between LRH1 overexpression and intraperitoneal metastasis (Table 3). Univariate analyses of clinicopathologic variables for intraperitoneal metastasis showed that the presence of intraperitoneal metastasis was positively associated with FIGO stage $(P<0.001)$, residual disease $(P=0.025)$, and ascites $(P=0.019)$. Multivariate logistic regression analysis revealed that FIGO stage $(P=0.003)$, residual disease $(P=0.032)$, and LRH1 $(P=0.010)$ were independently linked with intraperitoneal metastasis.

Results showed that the presence of lymph node metastasis was significantly correlated with histologic grade $(P=0.016)$ and LRH1 expression $(P=0.011)$. Multivariate logistic regression showed that histologic grade $(P=0.026)$ and LRH1 expression $(P=0.019)$ significantly led to lymph node metastasis (Table 4).

\section{LRH1 knockdown inhibits OC cell proliferation and migration in vitro}

LRH1 knockdown assays using sh-RNA was conducted to investigate the biological function of LRH1 in OC cells. We used RT-qPCR and Western blotting to confirm that the LRH1 expression in OVCAR3 and SKOV3 cells transfected with sh-LRH1 was remarkably lower than in those transfected with sh-NC (Figures 4B and C). We explored the effect of LRH1 knockdown on the proliferation of OVCAR3 and SKOV3 cells. The proliferation of OVCAR3 and SKOV3 cells infected with sh-LRH1 was repressed at 24, 48, 72, and 96 hours, respectively, compared with OVCAR3 and SKOV3 cells infected with sh-NC (Figure 4D). Thus, LRH1 knockdown substantially repressed cell proliferation.

We also investigated the effect of LRH1 on cell migration. Epithelial-mesenchymal transition (EMT) plays a crucial role in tumor invasion and metastasis in OC. We used Western blot to detect the protein levels of EMT markers, namely E-cadherin, N-cadherin, vimentin, and $\beta$-catenin. The results showed that E-cadherin 
and $\beta$-catenin level increased in LRH1 knockdown cells, whereas $\mathrm{N}$-cadherin and vimentin decreased. Moreover, transwell assay was conducted to evaluate cell migration. Migrated cells were counted in three random fields in transwell plates, and the average value of the three fields was calculated. The result of the transwell assay showed that knockdown of LRH1 decreased the migration of SKOV3 and OVCAR3 cells by approximately $42 \%$ and $50 \%$, respectively (Figure 4F). Wound-healing assay showed that LRH1 promoted the migration of SKOV3 and OVCAR3 cells (Figure 4G). After 24h, control SKOV3 and OVCAR3 cells migrated approximately 20 and $15 \mathrm{~mm}$, respectively, whereas SKOV3 and OVCAR3 cells with LRH1 knockdown migrated approximately 13 and $6 \mathrm{~mm}$, respectively. The cells with LRH1 knockdown showed less motility than control cells; thus, LRH1 could stimulate cell migration.

\section{Functional enrichment analysis of LRH1 in OC}

We obtained 230 genes that have the same expression pattern as LRH1 through Gene Expression Profiling Interactive Analysis as described in the Method section to evaluate the function of LRH1 in OC. We aimed to explain the mechanisms of LRH1 OC to determine the biological functions of these similar genes. We uploaded the 230 similar genes into Metascape and conducted custom analysis. As shown in Figures $5 \mathrm{~A}$ and $\mathrm{B}$, the function of these similar genes was related to the polycomb repressive complex 1 (PRC1 complex), the negative regulation of cell differentiation, methylation-dependent chromatin silencing, developmental growth, nephron tubule morphogenesis, ammonium ion binding, the negative regulation of neuron apoptotic process, mRNA mRNA 3' untranslated region (UTR) binding, homophilic cell adhesion via plasma membrane adhesion molecules, neuropeptide signaling pathway, the regulation of lipid transport, sodium ion transport, pattern specification process, and nucleosome assembly. Moreover, we applied Metascape to explore the protein-protein interactions of the genes similar to LRH1. As shown in Figures $\mathbf{5} \mathbf{C}$ and $\mathbf{D}$, the protein-protein interaction network was related to the PRC1 complex, nuclear ubiquitin ligase complex, and polycomb group (PcG) proteins.

\section{Discussion}

$\mathrm{OC}$ is the most lethal cancer among women, and its 5-year survival rate in advanced stages is $30 \%-40 \%$ [25]. The American Cancer Society reported that about 21,750 new cases of OC will be diagnosed and 13,940 women will die of OC in the United States in 2020 [1]. OC is a heterogeneous tumor that contains a heterogenous group of malignancies, which differ in etiology, molecular biology, and other characteristics [26]. High-risk OC has very low cure rates despite of therapy that comprised surgery, chemotherapy, PARPi, immunotherapy, and radiation [26]. Clearly, the identification of novel biomarkers and more effective therapies is extremely urgent.

LRH1 is a member of the nuclear receptor NR5A (Ftz-F1) subfamily, which is expresses in the endodermal tissues of the intestine, liver, exocrine pancreas [27], ovary [28-31], pre-adipocyte [32], and placenta [33]. Moreover, LRH1 plays an important role in a variety of biological functions, including cell proliferation, differentiation, and tumorigenesis. 
In the present study, we applied RT-qPCR to detect the mRNA level of LRH1 in patients with OC. Results showed that the increasing trend of LRH1 mRNA expression from $\mathrm{N}$ to $\mathrm{T} 1, \mathrm{~T} 2$, and its matched $\mathrm{M}$ (Figure 1). Moreover, we conducted immunohistochemical (IHC) assays to detect the protein level of LRH1. IHC revealed that $\mathrm{LRH} 1$ protein expression in normal tissues was scarcely detectable in normal tissues but intense in OC tissues (Figure 2). We also explored the relationship between LRH1 protein expression and clinicopathological factors and found that LRH1 predicts peritoneal metastasis and poor outcome. Therefore, LRH1 has a potential role as a biomarker of OC (Table1,2,3 and 4, Figure 3). Furthermore, the mRNA expression level of LRH1 was confirmed by UALCAN. Figure 4A shows that LRH1 mRNA expression was upregulated OC tissues in stages II and IV compared with those in stages I and III, but the difference was not statistically significant. In vitro assays showed that LRH1 could promote tumor cell proliferation, migration, and EMT (Figure 4D, E, F and G). Bioinformatics analysis was applied to investigate the biological function of LRH. Results demonstrated that the functions of LRH1 are associated with the PRC1 complex, the negative regulation of cell differentiation, methylation-dependent chromatin silencing, developmental growth, nephron tubule morphogenesis, ammonium ion binding, negative regulation of neuron apoptotic process, mRNA 3' UTR binding, homophilic cell adhesion via plasma membrane adhesion molecules, neuropeptide signaling pathway, lipid transport regulaton, sodium ion transport, pattern specification process, and nucleosome assembly (Figure 5).

The protein and mRNA levels of LRH1 are upregulated in various cancers [15-17], We found the same conclusion in our results. Lin et al. viewed LRH1 as a remrkable prognosis biomarker for tumor invasion and proliferation in pancreas cancer [34]. Thus, we were prompted to identify the role of LRH1 overexpression as a predictor of clinicopathological and prognostic relevance. We recognized that elevated LRH1 protein expression was strongly associated with FIGO stage, lymph node metastasis, and intraperitoneal metastasis. Furthermore, multivariate logistic regression model demonstrated that high LRH1 expression was remarkably associated with lymph node and intraperitoneal metastases. In addition, the OS periods of patients with high LRH1 expression were shorter than those of patients with low LRH1 expression. The patients with low LRH1 expression presented better prognoses than those with high LRH1 expression. The vitro assay of OC cells showed that LRH1 could promote tumor cell proliferation, migration, and EMT. Our findings are consistent with the results of previous research.

$\mathrm{LRH} 1$ protein functions as a cancer regulator that exists in extensive procedures, such as cell proliferation, chemotherapy resistance, and tumor progression $[12,13,35]$. The mechanism of LRH1 that influences migration demonstrated that LRH1 enhanced the transcriptional activity of $\beta$-catenin and upregulated the expression of downstream target genes (c-Myc and MMP2/9); thus, LRH1 promoted cell migration and invasion [14]. LRH1-induced chemotherapy resistance was reported in breast cancer. LRH1 promots the chemoresistance of breast cancer cells by enhancing the expression of MDC1 and attenuating DNA damage [13]. Bianco et al. found that LRH1 could increase the level of cyclin-dependent kinase inhibitor CDKN1A in a p53-independent manner, which results in tumor cell proliferation [36]. Bioinformatics analysis was applied to evaluate the function of $\mathrm{LRH} 1$ in $\mathrm{OC}$ and demonstrated that the functions of LRH1 are associated with the PRC1 complex, the negative regulation of cell differentiation, methylation-dependent chromatin silencing, developmental growth, nephron tubule morphogenesis, 
ammonium ion binding, the negative regulation of neuron apoptotic process, mRNA 3' UTR binding, homophilic cell adhesion via plasma membrane adhesion molecules, neuropeptide signaling pathway, lipid transport regulation, sodium ion transport, pattern specification process, and nucleosome assembly. These findings suggest the important biological role of LRH1 in carcinogenesis and tumor progression.

\section{Conclusion}

Although limitations still exist in this study, the results we observed showed that LRH1 contributes to lymph node metastasis, intraperitoneal metastasis, and poor prognosis in OC.Thus, LRH1 could be a specific biomarker for predicting poor prognosis and metastasis. Furthermore, LRH1 promotes tumor cell proliferation, migration and EMT in vitro. Bioinformatics analysis predicted that the functions of LRH1 are associated with PRC1 complex, nuclear ubiquitin ligase complex, and PcG proteins.

\section{Abbreviations}

LRH1: liver receptor homolog 1; OC: ovarian cancer; RT: reverse transcription; FIGO: the International Federation of Gynecology and Obstetrics; PBS: phosphate-buffered saline; IHC: Immunohistochemical; STR: short tandem repeat; SDS-PAGE: sodium dodecyl sulfate polyacrylamide gel electrophoresis; PVDF: polyvinylidene difluoride; OS: Overall survival; DFS: disease-free survival; EMT: epithelial-mesenchymal transition; PRC1 complex: polycomb repressive complex 1; UTR: untranslated region;

\section{Declarations}

\section{Authors' contributions:}

YLB conceived and designed the study. LJX and LJM collected samples and processed data. SQT provided technical support. SWZ and YLB analyzed data. SWZ drafted the manuscript. YLB revised the manuscript. All authors read and approved the final manuscript.

Acknowledgements: Not applicable.

\section{Competing interests:}

The authors declare that they have no competing interests.

\section{Availability of data and materials:}

All data generated and analyzed during this study are included in this published article and its additional file.

\section{Consent for publication:}

Not applicable. 


\section{Ethics approval and consent to participate:}

The current study was approved by the Ethics Committee of Harbin Medical University Cancer Hospital. A written consent from each participant was obtained after they were informed the purpose of this study.

\section{Funding:}

This study was supported by the Science and Technology Project of Education Department of Heilongjiang Province (12531275).

\section{References}

1. Cancer Stat Facts: Ovarian Cancer [https://seer.cancer.gov/statfacts/html/ovary.html

2. Colombo N, Sessa C, du Bois A, et al (2019) ESMO-ESGO Consensus Conference Recommendations on Ovarian Cancer: Pathology and Molecular Biology, Early and Advanced Stages, Borderline Tumours and Recurrent Diseaset. Ann Oncol 30(5):672-705.

3. Lheureux S, Gourley C, Vergote I, et al (2019) Epithelial ovarian cancer. Lancet 393(10177):12401253.

4. Sara Moufarrij, Monica Dandapani, Elisa Arthofer, et al (2019) Epigenetic Therapy for Ovarian Cancer: Promise and Progress. Clin Epigenetics 11(1):7.

5. Franzese E, Centonze S, Diana A, et al (2019) PARP Inhibitors in Ovarian Cancer. Cancer Treat Rev 73:1-9.

6. Antonio González-Martín, Bhavana Pothuri, Ignace Vergote, et al (2019) Niraparib in Patients With Newly Diagnosed Advanced Ovarian Cancer. N Engl J Med 381(25):2391-2402.

7. J Mateo, C J Lord, V Serra, et al (2019) A Decade of Clinical Development of PARP Inhibitors in Perspective. Ann Oncol 30(9):1437-1447.

8. Federica Tomao, Erlisa Bardhi, Anna Di Pinto, et al (2019) Parp Inhibitors as Maintenance Treatment in Platinum Sensitive Recurrent Ovarian Cancer: An Updated Meta-Analysis of Randomized Clinical Trials According to BRCA Mutational Status. Cancer Treat Rev 80:101909.

9. Sara Bouberhan, Eric Pujade-Lauraine, Stephen A Cannistra (2019) Advances in the Management of Platinum-Sensitive Relapsed Ovarian Cancer. J Clin Oncol37(27):2424-2436.

10. Fayard E, Auwerx J, Schoonjans K (2004) LRH-1: an orphan nuclear receptor involved in development, metabolism and steroidogenesis. Trends in cell biology 14(5):250-60.

11. Lee YK, Moore DD (2008) Liver receptor homolog-1, an emerging metabolic modulator. Frontiers in bioscience : a journal and virtual library 13:5950-8.

12. Jia-Min B Pang, Ramyar Molania, Ashwini Chand, et al (2017) LRH-1 Expression Patterns in Breast Cancer Tissues Are Associated With Tumour Aggressiveness. Oncotarget 8(48):83626-83636.

13. Wang S, Zou Z, Luo X, et al (2018) LRH1 enhances cell resistance to chemotherapy by transcriptionally activating MDC1 expression and attenuating DNA damage in human breast cancer. 
Oncogene 37(24):3243-59.

14. Lin Q, Aihara A, Chung W, et al (2014) LRH1 promotes pancreatic cancer metastasis. Cancer Lett 350(1-2):15-24.

15. Wu C, Feng J, Li L, et al (2018) Liver receptor homologue 1, a novel prognostic marker in colon cancer patients. Oncol Lett 16(3):2833-8.

16. Liu $Y$, Xing $Y$, Wang $H$, et al (2018) LRH1 as a promising prognostic biomarker and predictor of metastasis in patients with non-small cell lung cancer. Thorac Cancer 9(12):1725-32.

17. Jin J, Jin J, Woodfield SE, et al (2019) Targeting LRH1 in hepatoblastoma cell lines causes decreased proliferation. Oncol Rep 41(1):143-53.

18. Benedet JL, Bender $\mathrm{H}$, Jones $\mathrm{H}$, et al (2000) FIGO staging classifications and clinical practice guidelines in the management of gynecologic cancers. FIGO Committee on Gynecologic Oncology. International journal of gynaecology and obstetrics: the official organ of the International Federation of Gynaecology and Obstetrics 70(2):209-62.

19. Penzel R, Hoegel J, Schmitz W, et al (2003) Clusters of chromosomal imbalances in thymic epithelial tumours are associated with the WHO classification and the staging system according to Masaoka. International journal of cancer 105(4):494-8.

20. Labile Togba Soumaoro, Hiroyuki Uetake, Tetsuro Higuchi, et al (2004) Cyclooxygenase-2 Expression: A Significant Prognostic Indicator for Patients With Colorectal Cancer. Clin Cancer Res10(24):846571.

21. Livak KJ, Schmittgen TD (2001) Analysis of relative gene expression data using real-time quantitative PCR and the 2(-Delta Delta C(T)) method. Methods 25: 402-8.

22. Darshan S Chandrashekar, Bhuwan Bashel, Sai Akshaya Hodigere Balasubramanya, et al (2017) UALCAN: A Portal for Facilitating Tumor Subgroup Gene Expression and Survival Analyses. Neoplasia 19(8):649-658.

23. Zefang Tang, Chenwei Li, Boxi Kang, et al (2017) GEPIA: A Web Server for Cancer and Normal Gene Expression Profiling and Interactive Analyses. Nucleic Acids Res 45(W1):W98-W102.

24. Yingyao Zhou, Bin Zhou, Lars Pache, et al (2019) Metascape Provides a Biologist-Oriented Resource for the Analysis of Systems-Level Datasets. Nat Commun 10(1):1523.

25. Ben Davidson, Claes G Tropé (2014) Ovarian Cancer: Diagnostic, Biological and Prognostic Aspects. Womens Health (Lond) 10(5):519-33.

26. Torre LA, Trabert B, DeSantis CE, et al (2018) Ovarian cancer statistics, 2018. CA: a cancer journal for clinicians 68(4):284-96.

27. Lu TT, Repa JJ, Mangelsdorf DJ (2001) Orphan nuclear receptors as eLiXiRs and FiXeRs of sterol metabolism. The Journal of biological chemistry 276(41):37735-8.

28. Boerboom D, Pilon N, Behdjani R, et al (2000) Expression and regulation of transcripts encoding two members of the NR5A nuclear receptor subfamily of orphan nuclear receptors, steroidogenic factor- 1 and NR5A2, in equine ovarian cells during the ovulatory process. Endocrinology 141(12):4647-56. 
29. Schoonjans K, Annicotte JS, Huby T, et al (2002) Liver receptor homolog 1 controls the expression of the scavenger receptor class B type I. EMBO reports 3(12):1181-7.

30. Hinshelwood MM, Repa JJ, Shelton JM, et al (2003) Expression of LRH-1 and SF-1 in the mouse ovary: localization in different cell types correlates with differing function. Molecular and cellular endocrinology 207(1-2):39-45.

31. Clyne CD, Speed CJ, Zhou J, et al (2002) Liver receptor homologue-1 (LRH-1) regulates expression of aromatase in preadipocytes. The Journal of biological chemistry 277(23):20591-7.

32. Liu DL, Liu WZ, Li QL, et al (2003) Expression and functional analysis of liver receptor homologue 1 as a potential steroidogenic factor in rat ovary. Biology of reproduction 69(2):508-17.

33. Sirianni R, Seely JB, Attia G, et al (2002) Liver receptor homologue-1 is expressed in human steroidogenic tissues and activates transcription of genes encoding steroidogenic enzymes. The Journal of endocrinology 174(3):R13-7.

34. Lin Q, Aihara A, Chung W, et al (2014) LRH1 as a driving factor in pancreatic cancer growth. Cancer Lett 345(1):85-90.

35. Li C, Dong J, Han Z, Zhang K (2017) MicroRNA-219-5p Represses the Proliferation, Migration, and Invasion of Gastric Cancer Cells by Targeting the LRH-1/Wnt/ $\beta$-Catenin Signaling Pathway. Oncology research 25(4):617-27.

36. S Bianco, M Jangal, D Garneau, et al (2015) LRH-1 Controls Proliferation in Breast Tumor Cells by Regulating CDKN1A Gene Expression. Oncogene34(34):4509-18.

\section{Tables}


Table 1

Correlation between LRH1 protein expression level and clinicopathological variables in 133 patients with OC

\begin{tabular}{|c|c|c|c|c|}
\hline \multirow[t]{2}{*}{ Variables } & \multirow{2}{*}{$\begin{array}{l}\text { All patients } \\
\mathrm{n}=133\end{array}$} & \multicolumn{2}{|c|}{ LRH1 expression level } & \multirow[t]{2}{*}{$P$} \\
\hline & & $\begin{array}{l}\text { Low }(\%) \\
(n=59)\end{array}$ & $\begin{array}{l}\text { High (\%) } \\
(n=74)\end{array}$ & \\
\hline Age (years) & 67 & $33(49.3)$ & $34(50.7)$ & 0.252 \\
\hline$\leq 52$ & 66 & $26(39.4)$ & $40(60.6)$ & 0.653 \\
\hline$>52$ & 92 & $42(45.7)$ & $50(54.3)$ & 0.001 \\
\hline Histological type & 41 & $17(41.5)$ & $24(58.5)$ & 0.310 \\
\hline Serous & 28 & $20(71.4)$ & $8(28.6)$ & 0.851 \\
\hline Others & 105 & $39(37.1)$ & $66(62.9)$ & 0.473 \\
\hline FIGO stage & 50 & $25(50.0)$ & $25(50.0)$ & 0.653 \\
\hline $1 / 11$ & 83 & $34(41.0)$ & $49(59.0)$ & 0.011 \\
\hline III/IV & 98 & $43(43.9)$ & $55(56.1)$ & 0.001 \\
\hline Histologic grade & 35 & $16(45.7)$ & $19(54.3)$ & \\
\hline G1/G2 & 38 & $15(39.5)$ & $23(60.5)$ & \\
\hline G3 & 95 & $44(46.3)$ & $51(53.7)$ & \\
\hline Residual disease $(\mathrm{cm})$ & 41 & $17(41.5)$ & $24(58.5)$ & \\
\hline$k 1$ & 92 & $42(45.7)$ & $50(54.3)$ & \\
\hline$\geq 1$ & 81 & $43(53.1)$ & $38(46.9)$ & \\
\hline Ascites (ml) & 52 & $16(30.8)$ & $36(69.2)$ & \\
\hline$<100$ & 43 & $28(65.1)$ & $15(34.9)$ & \\
\hline$\geq 100$ & 90 & $31(34.4)$ & $59(65.6)$ & \\
\hline \multicolumn{5}{|c|}{ Serum CA-125 level (U/ml) } \\
\hline \multicolumn{5}{|l|}{$\leq 35$} \\
\hline
\end{tabular}




\begin{tabular}{|c|c|c|c|c|}
\hline \multirow[t]{2}{*}{ Variables } & \multirow{2}{*}{$\begin{array}{l}\text { All patients } \\
\mathrm{n}=133\end{array}$} & \multicolumn{2}{|c|}{ LRH1 expression level } & $P$ \\
\hline & & $\begin{array}{l}\text { Low }(\%) \\
(n=59)\end{array}$ & $\begin{array}{l}\text { High }(\%) \\
(n=74)\end{array}$ & \\
\hline \multicolumn{5}{|c|}{$>35$} \\
\hline \multicolumn{5}{|c|}{ Lymph node metastasis } \\
\hline \multicolumn{5}{|l|}{ No } \\
\hline \multicolumn{5}{|l|}{ Yes } \\
\hline \multicolumn{5}{|c|}{ Peritoneum metastasis } \\
\hline \multicolumn{5}{|l|}{ No } \\
\hline Yes & & & & \\
\hline
\end{tabular}


Table 2

Univariate and multivariate analyses for survival in $133 \mathrm{OC}$ patients

\begin{tabular}{|c|c|c|c|}
\hline \multirow[t]{2}{*}{ Variables } & Univariate & \multicolumn{2}{|l|}{ Multivariate } \\
\hline & $P$ & $\mathrm{HR} 95 \% \mathrm{Cl} P$ & \\
\hline variables related to OS & 0.219 & $3.695(1.793-7.613)$ & $<0.001$ \\
\hline Age (years) & 0.914 & $1.664(1.101-2.513)$ & 0.016 \\
\hline Histological type & $<0.001$ & $1.682(1.093-2.591)$ & 0.018 \\
\hline FIGO stage & 0.009 & $3.665(2.344-5.730)$ & $<0.001$ \\
\hline Histologic grade & 0.022 & $3.437(1.641-7.200)$ & 0.001 \\
\hline Residual disease $(\mathrm{cm})$ & 0.696 & $1.550(1.024-2.345)$ & 0.038 \\
\hline Ascites (ml) & 0.154 & $1.663(1.065-2.597)$ & 0.025 \\
\hline Serum CA-125 level (U/ml) & $<0.001$ & $3.857(2.431-6.120)$ & $<0.001$ \\
\hline Lymph node metastasis & 0.001 & $1.535(1.017-2.318)$ & 0.041 \\
\hline Peritoneum metastasis & 0.007 & & \\
\hline LRH1 expression level & 0.487 & & \\
\hline variables related to DFS & 0.895 & & \\
\hline Age (years) & $<0.001$ & & \\
\hline Histological type & 0.028 & & \\
\hline FIGO stage & 0.026 & & \\
\hline Histologic grade & 0.855 & & \\
\hline Residual disease $(\mathrm{cm})$ & 0.281 & & \\
\hline Ascites (ml) & $<0.001$ & & \\
\hline Serum CA-125 level (U/ml) & $<0.001$ & & \\
\hline Lymph node metastasis & $<0.001$ & & \\
\hline \multicolumn{4}{|l|}{ Peritoneum metastasis } \\
\hline LRH1 expression level & & & \\
\hline
\end{tabular}


Page $17 / 25$ 
Table 3

Univariate and multivariable analyses for the relationship between LRH1 expression and lymph node metastasis in OCs

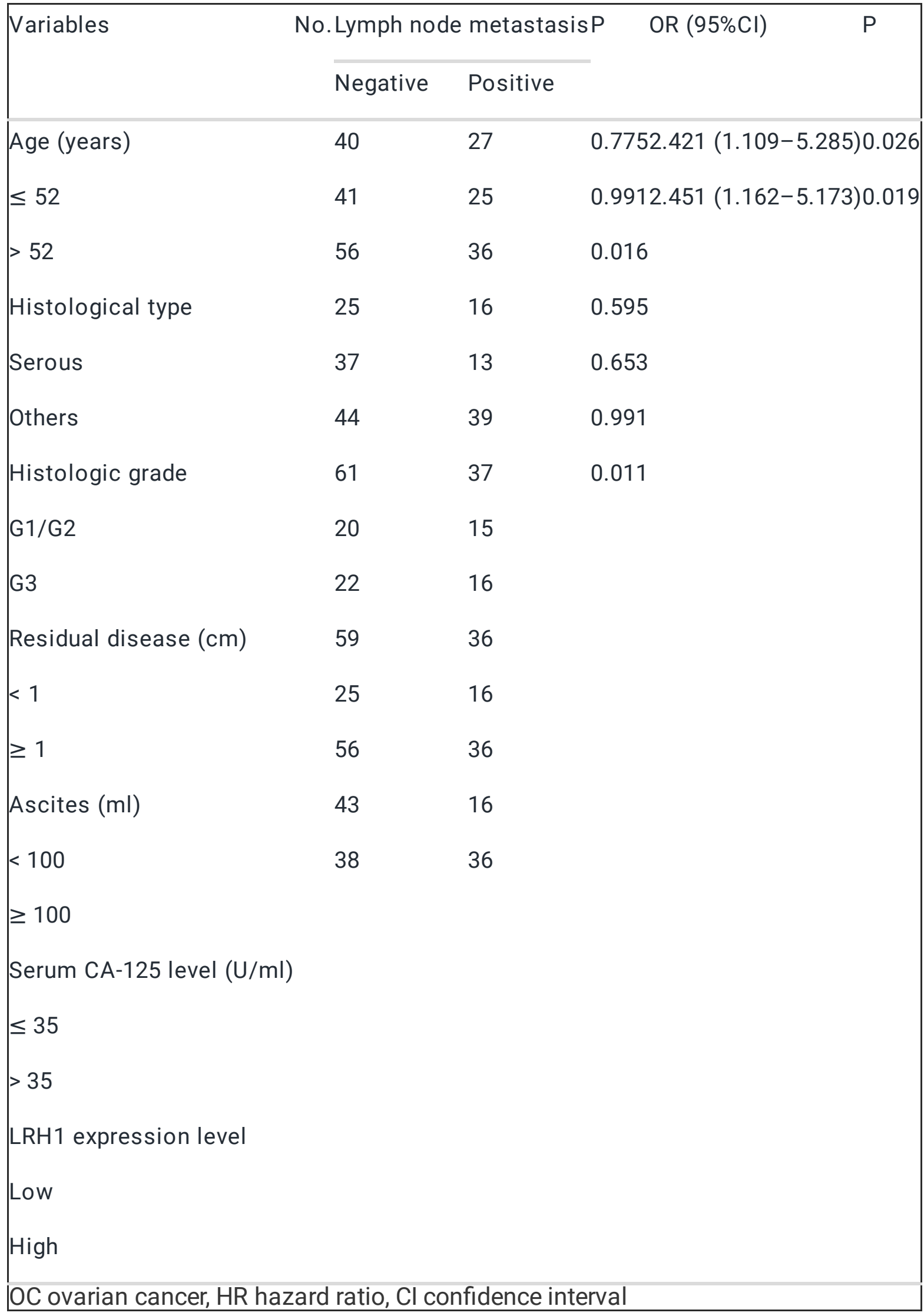


Page 19/25 
Table 4

Univariate and multivariable analyses for the relationship between LRH1 expression and peritoneum metastasis in OCs

\begin{tabular}{|c|c|c|c|c|c|}
\hline \multirow[t]{2}{*}{ Variables } & \multicolumn{2}{|c|}{ No. Peritoneum metastasis } & \multirow[t]{2}{*}{$\mathrm{P}$} & \multirow[t]{2}{*}{ OR $(95 \% \mathrm{Cl})$} & \multirow[t]{2}{*}{$\mathrm{P}$} \\
\hline & Negative & Positive & & & \\
\hline Age (years) & 22 & 45 & 0.900 & $4.613(1.678-12.677)$ & 0.003 \\
\hline$\leq 52$ & 21 & 45 & 0.918 & $3.208(1.104-9.322)$ & 0.032 \\
\hline$>52$ & 30 & 62 & $<0.001$ & $3.160(1.323-7.545)$ & 0.010 \\
\hline Histological type & 13 & 28 & 0.064 & & \\
\hline Serous & 19 & 9 & 0.025 & & \\
\hline Others & 24 & 81 & 0.019 & & \\
\hline FIGO stage & 21 & 29 & 0.614 & & \\
\hline $\mid / I 1$ & 22 & 61 & 0.001 & & \\
\hline III/IV & 37 & 61 & & & \\
\hline Histologic grade & 6 & 29 & & & \\
\hline G1/G2 & 18 & 20 & & & \\
\hline G3 & 25 & 70 & & & \\
\hline Residual disease $(\mathrm{cm})$ & 12 & 29 & & & \\
\hline$<1$ & 31 & 61 & & & \\
\hline$\geq 1$ & 28 & 31 & & & \\
\hline Ascites (ml) & 15 & 59 & & & \\
\hline \multicolumn{6}{|l|}{$<100$} \\
\hline \multicolumn{6}{|l|}{$\geq 100$} \\
\hline \multicolumn{6}{|c|}{ Serum CA-125 level (U/ml) } \\
\hline \multicolumn{6}{|l|}{$\leq 35$} \\
\hline \multicolumn{6}{|l|}{$>35$} \\
\hline $\begin{array}{l}\text { FIGO the Federation o } \\
\text { interval }\end{array}$ & gy and Obs & 0 & $a$ & 21 & e \\
\hline
\end{tabular}




\begin{tabular}{|c|c|c|c|c|}
\hline \multirow[t]{2}{*}{ Variables } & \multicolumn{2}{|c|}{ No. Peritoneum metastasis } & \multirow[t]{2}{*}{ OR $(95 \% \mathrm{Cl})$} & \multirow[t]{2}{*}{$\mathrm{P}$} \\
\hline & Negative & Positive & & \\
\hline \multicolumn{5}{|c|}{ LRH1 expression level } \\
\hline \multicolumn{5}{|l|}{ Low } \\
\hline \multicolumn{5}{|l|}{ High } \\
\hline
\end{tabular}

\section{Figures}

A

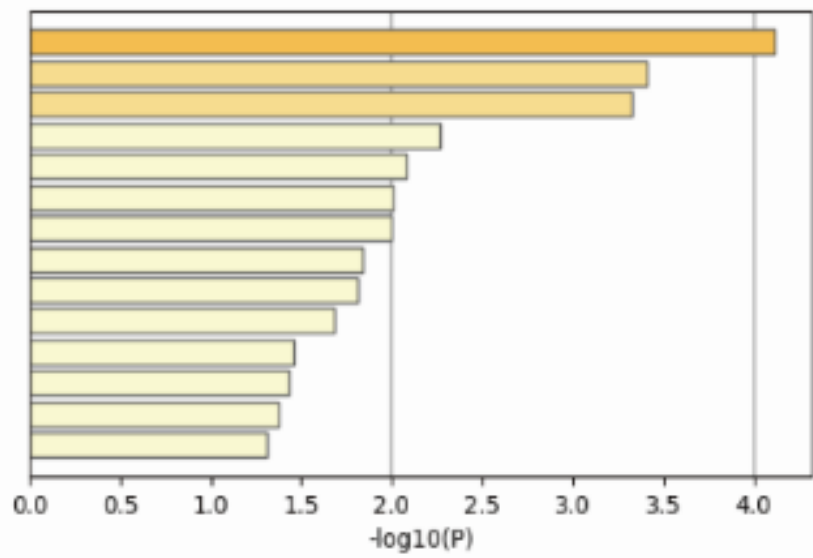

B

GO:0035102: PRC1 complex

G0:0045596: negative regulation of cell differentiation

GO:0006346: methylation-dependent chromatin silencing

GO:0048589: developmental growth

GO:0072078: nephron tubule morphogenesis

GO:0070405: ammonium ion binding

GO:0043524: negative regulation of neuron apoptotic process

GO:0003730: mRNA 3'-UTR binding

GO:0007156: homophilic cell adhesion via plasma membrane adhesion molecules

G0:0007218: neuropeptide signaling pathway

GO:0032368: regulation of lipid transport

GO:0006814: sodium ion transport

GO:0007389: pattern specification process

G0:0006334: nucleosome assembly
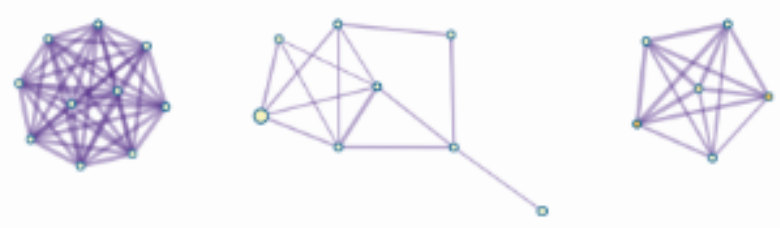

C
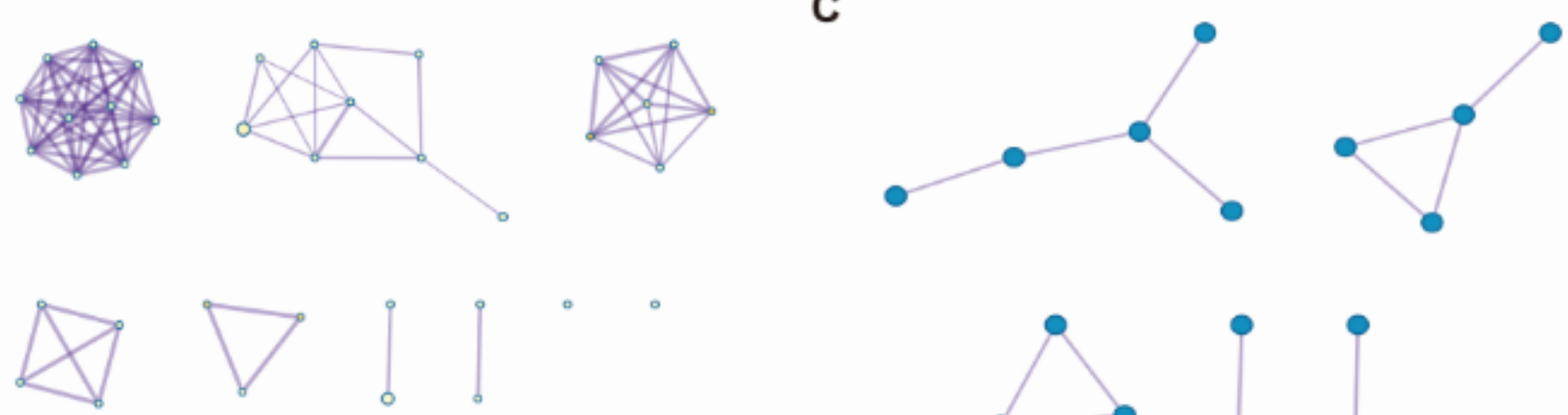

$\circ$

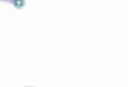

D

\begin{tabular}{ll} 
GO & Description \\
\hline GO:0035102 & PRC1 complex \\
GO:0000152 & nuclear ubiquitin ligase complex \\
GO:0031519 & PCG protein complex
\end{tabular}
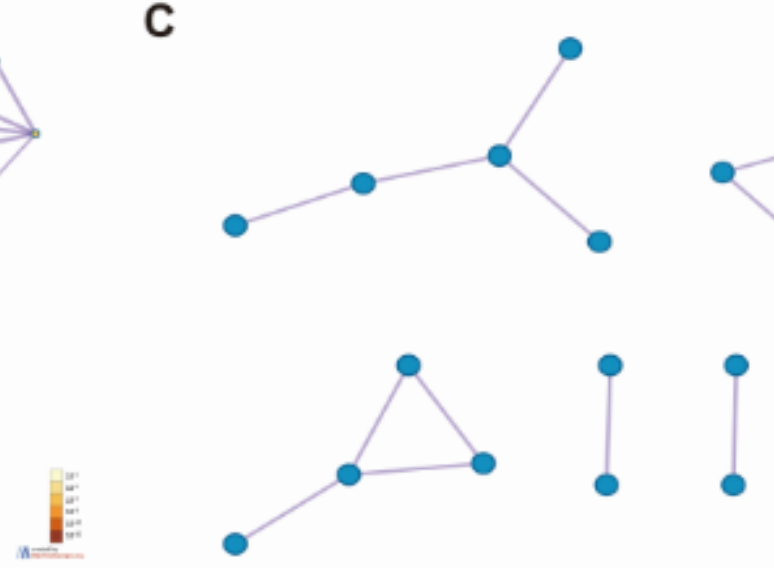

E

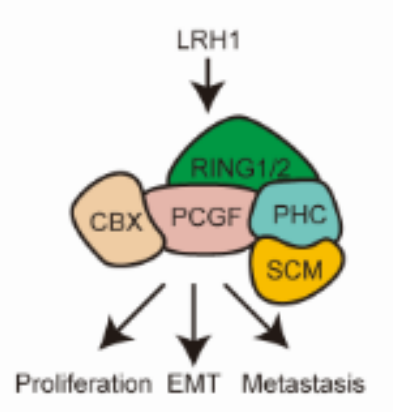


Figure 1

Functional enrichment analysis of genes that have similar expression pattern to LRH1 in ovarian cancer (Metascape). (A) Heatmap of Gene Ontology (GO) and enriched terms colored by p-values. (B) Network of GO enriched terms colored by p-value. (C) Protein-protein interaction network and MCODE components identified in the gene lists. (D) Independent functional enrichment analysis of MCODE components. (E) A schematic model depicting LRH1 upregulation probably promotes cell proliferation, EMT, metastasis by activating PRC1 complex in ovarian cancer cells via bioinformation analysis.

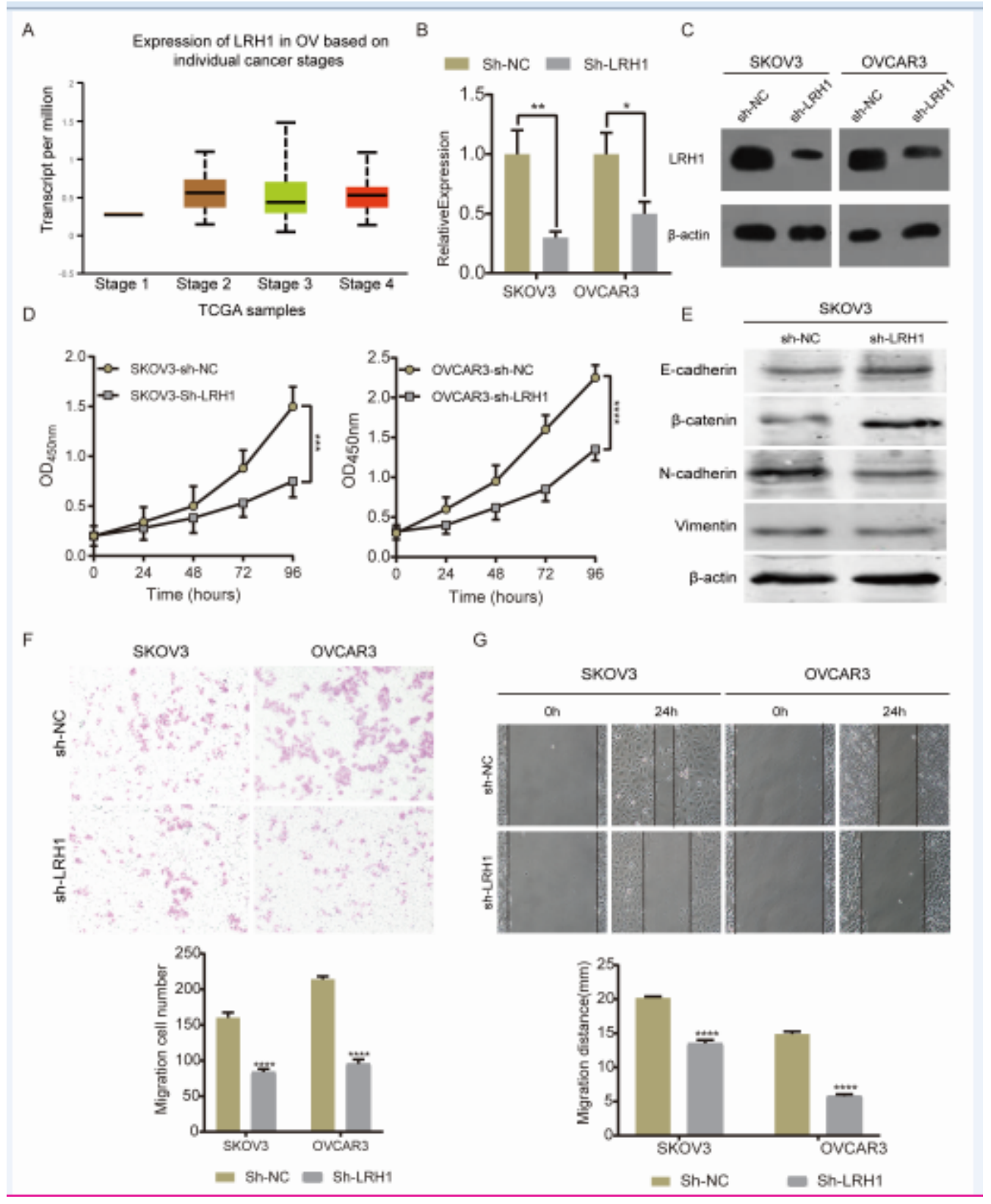

Figure 2

Knockdown of LRH1 inhibits ovarian cancer cells proliferation and migration in vitro. (A) The mRNA expression of LRH1 in ovarian cancer (UALCAN). The level of mRNA (B) and protein (C) expression significantly decreased after sh-LRH1 transfection. CCK8 assays (D) have revealed that knockdown of 
LRH1 inhibits cell proliferation. (E) Western blotting was applied to detect E-cadherin, $\beta$-catenin, $\mathrm{N}$ cadherin, vimentin and $\beta$-actin with the respective antibodies. Transwell assays $(F)$ and wound healing assays $(G)$ have indicated that knockdown of LRH1 inhibits cell migration. ${ }^{* \star \star *} \mathrm{P}<0.0001$, ${ }^{\star \star *} \mathrm{P}<0.001$, $\star * P<0.01, * P<0.05$; data are shown as the mean \pm SD.

A

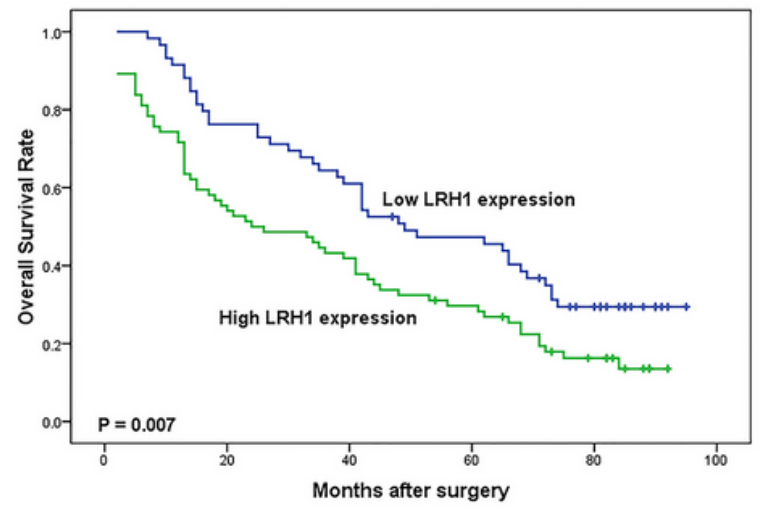

B

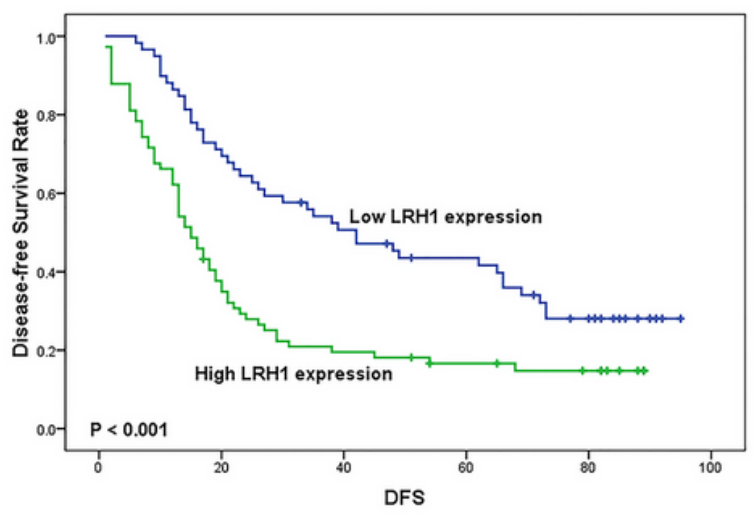

\section{Figure 3}

Kaplan-Meier analysis of overall survival (OS) and disease-free survival (DFS) related to the expression of LRH1. A OS curves of the $133 \mathrm{OC}$ patients; B DFS curves of the $133 \mathrm{OC}$ patients. 

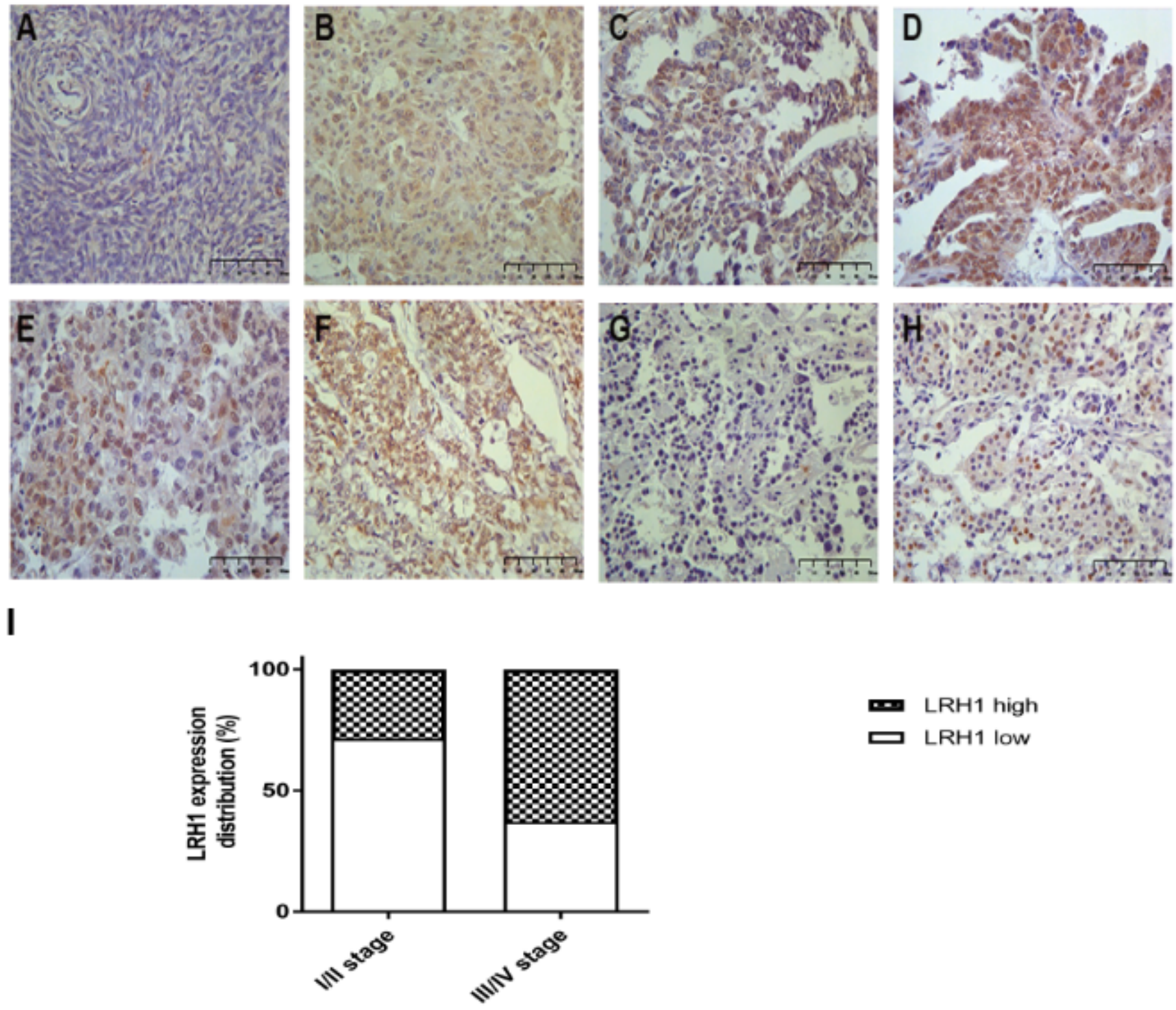

Figure 4

Immunohistochemical anlaysis of LRH1 protein expression (x 400). A negative or weak expression in normal ovarian tissues; $B$ high expression in primary ovarian cancer $(O C)$ tissues (observed mainly in nucleus, a few in cytoplasmic); C low expression in serous histotypes (mainly in nucleus); D high expression in serous histotypes (mainly in nucleus, a few in cytoplasmic); $E$ high expression in mucinous histotypes (mainly in nucleus); $F$ high expression in endometrioid histotypes (mainly in nucleus, a few in cytoplasmic); $\mathrm{G}$ negative or low expression in clear cell histotypes; $\mathrm{H}$ high expression in clear cell histotypes (mainly in nucleus, scarcely in cytoplasmic); I the status of LRH1 expression in different FIGO stages. 


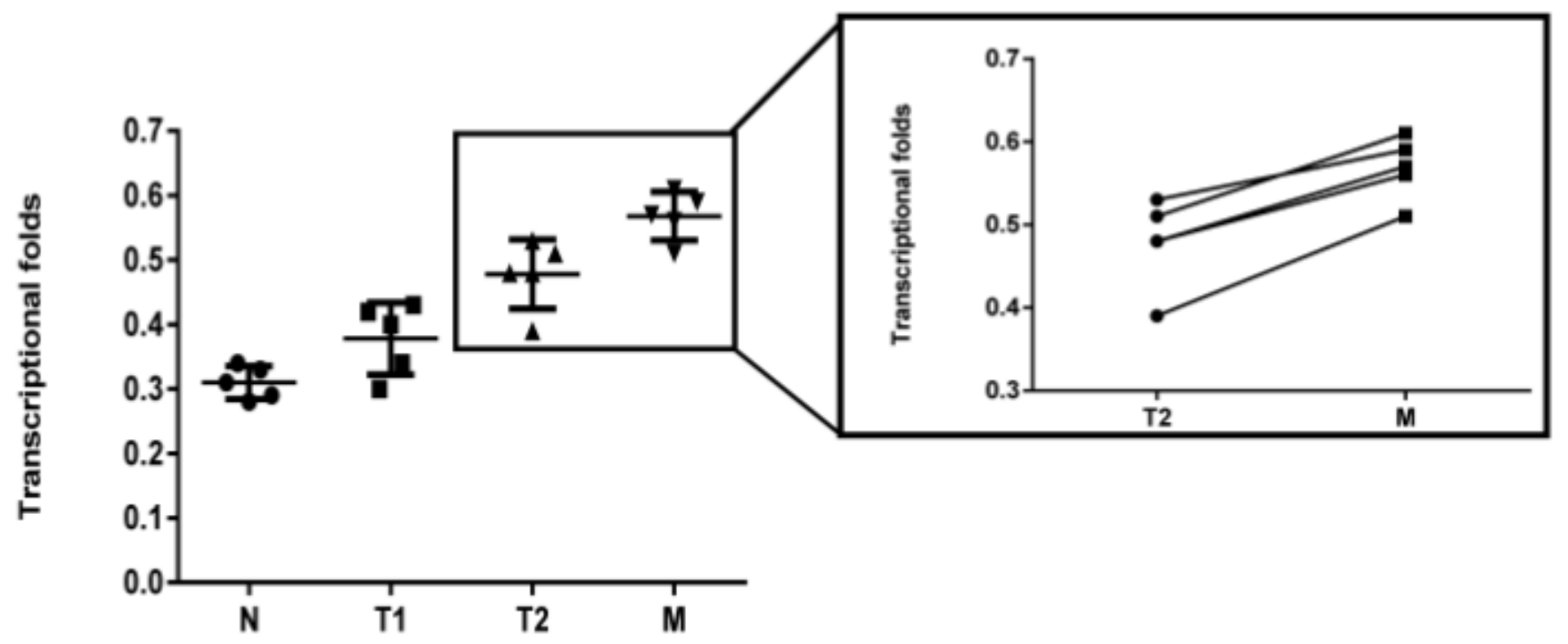

Figure 5

Real time PCR assay of LRH1 mRNA expression in N, T1, T2, and its matched $\mathrm{M}$. It showed that elevated trend of LRH1 mRNA expression from $\mathrm{N}$ to $\mathrm{T} 1, \mathrm{~T} 2$ and its matched $\mathrm{M}(\mathrm{P}<0.05)$. 